\title{
Das schöne, glatte Bein: Haarentfernung durch Laser und lichtbasierte Verfahren
}

\section{Light-based hair removal technologies for beautiful legs}

Autoren

Tatjana Braun, Pia Kleimann, Markus Meissner, Roland Kaufmann, Eva Maria Valesky

Institut

Klinik für Dermatologie, Venerologie und Allergologie, Universitätsklinikum Frankfurt, Goethe-Universität Frankfurt, Deutschland

Schlüsselwörter

Fotoepilation, Intense pulsed light device (IPL), Melanin, Haare

Key words

light based hair removal, intense pulsed light device (IPL), melanin, hair

online publiziert 20.05 .2021

Bibliografie

Phlebologie 2021; 50: 324-329

DOI 10.1055/a-1187-1932

ISSN 0939-978X

(c) 2021. Thieme. All rights reserved.

Georg Thieme Verlag KG, Rüdigerstraße 14,

70469 Stuttgart, Germany

Korrespondenzadresse

Prof. Dr. Eva Maria Valesky

Klinik für Dermatologie, Venerologie und Allergologie

Universitätsklinikum Frankfurt, Goethe-Universität,

Theodor-Stern-Kai 7, 60590 Frankfurt am Main, Deutschland

Eva.Valesky@kgu.de

\section{ZUSAMMENFASSUNG}

Die Fotoepilation zählt zu den meistgefragten nichtchirurgischen Eingriffen in der ästhetischen Dermatologie. Haarlose, glatte Beine gelten dabei als Schönheitsideal.

Fotoepilation mittels Laser oder hochenergetischer Blitzlampen (sog. Intensed-Pulsed-Light-Geräte) stellt ein sicheres und effektives Verfahren dar. Der Wirkmechanismus beruht auf dem Prinzip der selektiven Fotothermolyse, wobei eine gezielte thermische Schädigung pigmentierter Haarfollikel unter Schonung des umliegenden Gewebes erfolgt. Melaninpigment des Haarschaftes absorbiert als Zielchromophor Licht eines speziellen Wellenlängenspektrums und führt durch Wärmediffusion zur Schädigung der für das Haarwachstum wichtigen Stammzellen im äußeren Haarwurzelschaft. Patienten müssen über die Notwendigkeit wiederholter Behandlungen sowie den häufig nur partiellen und temporären Haarverlust aufgeklärt werden. Dunkle Terminalhaare bei hellen Hauttypen lassen sich effektiv entfernen. Bei dunklen Hauttypen besteht aufgrund des erhöhten epidermalen Melaninvorkommens ein erhöhtes Risiko für Nebenwirkungen wie Verbrennungen, Dyspigmentierungen und Narben. Sehr feine, helle oder rote Haare lassen sich kaum mit konventionellen Fotoepilationsverfahren entfernen. Haarentfernungsgeräte für den Heimgebrauch sind eine kostengünstige, einfach anzuwendende Alternative, weisen aber im Vergleich zur professionellen Fotoepilation eine geringere Effektivität auf.

\section{ABSTRACT}

Light-based hair removal is one of the most frequently requested, non-invasive procedures in cosmetic dermatology, especially on the legs. Laser and intense pulsed light devices (IPL) allow for effective and safe hair removal. The mechanism of action is based on selective photothermolysis, causing selective thermal damage of pigmented hair follicles while sparing surrounding tissue. Melanin pigment in the hair shaft and matrix region serves as target chromophore and absorbs light of a specific wavelength spectrum, which is then converted to heat. Heat diffusion along the hair shaft causes damage to non-pigmented stem cells of the bulge region and thereby delays hair growth.

Patients require multiple treatments and hair removal is generally incomplete and only temporary. Dark terminal hair on light skin types can be removed very effectively by light-based hair removal methods. Complications such as burns, dyspigmentation and scaring are more frequent in darker skin types due to higher epidermal melanin concentrations. Very fine, light or red hair show low removal rates even with state-ofthe-art methods. Home-use of light-based devices pose a convenient, less expensive and easy to use alternative for hair removal. Efficacy, however, is lower compared to professional hair removal procedures. 


\section{Einleitung}

Die Entfernung ungewollter Körperbehaarung stellt bei Frauen und zunehmend auch bei Männern einen wachsenden Trend dar. Glatte, haarlose Beine gelten dabei in unserer heutigen Gesellschaft für Viele als erstrebenswertes Schönheitsideal.

Pathologischer, übermäßiger Haarwuchs kann sich in Form einer Hypertrichose oder an Androgen-abhängigen Arealen in Form eines Hirsutismus in unterschiedlichen Ausprägungsgraden als Symptom einer endokrinologischen Störung (ovarielle, adrenale oder zentrale Hormonanomalie) manifestieren sowie medikamentös induziert oder idiopathisch auftreten. Meist erfolgt die Haarentfernung allerdings aus rein kosmetischen Gründen ohne Vorliegen einer Grunderkrankung.

Zur kurzfristigen Enthaarung steht eine Vielzahl mechanischer (Rasur, Epilation, Wachsentfernung) oder chemischer Verfahren (bleichende oder Enthaarungsmittel) zur Auswahl. Die langfristige Reduktion der Haarfollikel wird durch Elektrolyse oder lichtbasierte Therapien (sog. Fotoepilation) ermöglicht.

Laser (englisches Akronym für light amplification by stimulated emission of radiation) und hochenergetische Blitzlampen (engl. intense pulsed light, IPL-Systeme) gelten seit den 90er-Jahren als Goldstandard für die Entfernung unerwünschten Haarwuchses. In Europa und den USA zählt die Fotoepilation zu den häufigsten nichtchirurgischen Eingriffen in der ästhetischen Dermatologie [1]. Die Beine werden dabei am häufigsten behandelt, gefolgt von Axillen, Gesicht und Genitalbereich [2].

Im Gegensatz zum monochromatischen Licht der Lasergeräte, welche Licht einer einzigen Wellenlänge bzw. eines schmalen Spektrums an Wellenlängen erzeugen, generieren IPL-Geräte ein breites Spektrum hochenergetischer, inkohärenter Lichtstrahlung im sichtbaren Licht bis Infrarot-Bereich (500-1200 nm). Je nach verwendeten Einstellungen und sog. Cut-off-Filtern wird dabei eine definierte Wellenlängenbreite emittiert, um die gewünschten Zielstrukturen zu erreichen.

\section{Wirkmechanismus}

Lichtbasierte Epilationsverfahren mittels Laser oder IPL-Systemen beruhen auf dem Prinzip der selektiven Fotothermolyse. Hierbei kommt es zu einer gezielten thermischen Schädigung pigmentierter Haarfollikel bei gleichzeitiger Schonung der umliegenden Gewebe. Das Melaninpigment des Haarschafts dient als Zielchromophor, welches Licht einer speziellen Wellenlänge absorbiert und dadurch erwärmt wird. Das vorhandene Melanin führt über Wärmediffusion zu einer Erhitzung des Haarschaftes und somit zu einer Eiweißdenaturierung der nichtpigmentierten Stammzellen im sog. Wulstbereich (engl. bulge area) im äußeren Wurzelschaft (ca. 1,5 mm unterhalb der Epidermis) sowie in der Matrixregion der dermalen Papille. So werden die Zielstrukturen geschädigt, die für das zyklische Haarwachstum notwendig sind $(\triangleright$ Abb. 1).

Die Energiedichte (engl. fluence, $\mathrm{J} / \mathrm{cm}^{2}$ ) und die Pulsdauer der Laser bzw. IPL-Geräte bestimmen die Energieabsorption durch

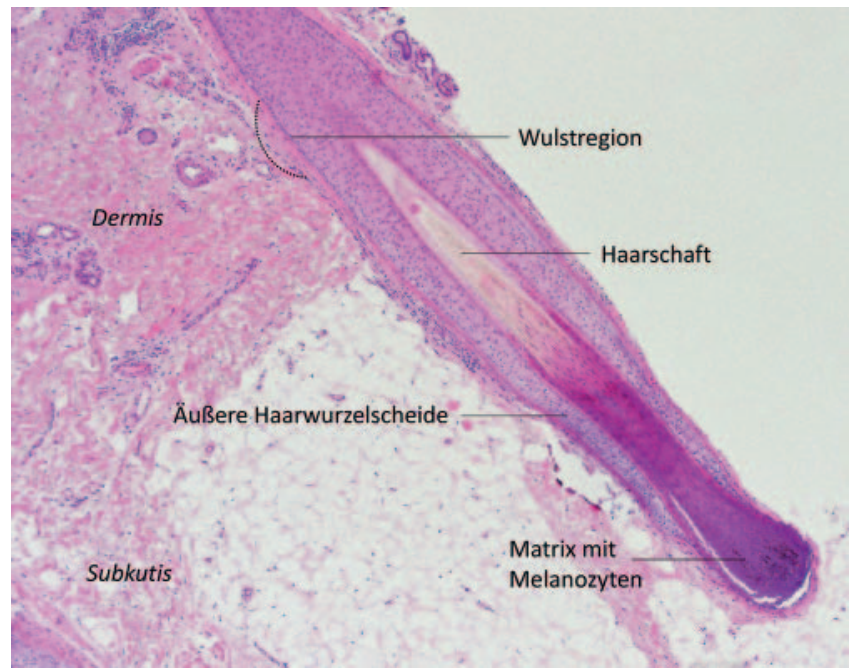

- Abb. 1 Histologischer Aufbau eines Haarfollikels mit Zielchromophor Melanin und der Stammzell-tragenden Wulstregion (HE-Schnitt, 100x Vergrößerung).

das Chromophor und damit die Wärmeentwicklung. Der selektivste thermische Schaden entsteht dabei, wenn die Pulsdauer kleiner oder gleich der thermalen Relaxationszeit von Melanin (thermal relaxation time, TRT) ist. Diese ist definiert als die Zeit, die eine Struktur benötigt, um auf die Hälfte der Zeit abzukühlen, die unmittelbar nach der Laserexposition erreicht wird. Für das menschliche Terminalhar variiert die TRT zwischen 10 und $100 \mathrm{~ms}$ [3].

Lang gepulste Laser arbeiten mit Impulsdauern im Millisekunden-Bereich, entsprechen daher annähernd der TRT der Terminalhaare und sind somit besonders gut für die Epilation geeignet. Durch Verwendung von Pulsdauern, die länger sind als die TRT der Haare, kann die Schädigung der Stammzellen im Haarschaft noch verstärkt werden (sog. Superlang gepulste Laser) [4].

Ein vollständiger Verlust der Haarfollikel wird pro Behandlung bei optimalen Einstellungen nur in ca. 15-30\% der behandelten Haare erreicht [5]. Eine kontrovers diskutierte Erklärung hierfür ist, dass der Grad der Pigmentierung sowie die Tiefenlokalisation der pigmentierten Haaranteile je nach Wachstumsphase des einzelnen Haares variieren und dadurch Zielstrukturen besonders effektiv nur in der Anagen-Phase (Wachstumsphase) durch Licht erreicht werden [5-7].

Entscheidend ist, dass meist nur ein temporärer Haarverlust durch Induktion einer Telogen-ähnlichen Ruhephase erzielt wird. Diese vorübergehende Alopezie dauert einige Wochen bis wenige Monate an, bis ein Großteil der Haare sich erholt und ein neuer Wachstumszyklus beginnt [8].

Nach 1-maliger Behandlung mit einem Dioden-Laser beispielsweise konnte gezeigt werden, dass die Wiederwachstumsrate der Haare nach einem Monat zwischen 22 und $31 \%$ betrug und schließlich im Zeitraum von 3-20 Monaten nach der Behandlung ein Plateau bei 65-75\% Wiederwachstumsrate erreichte [9]. 


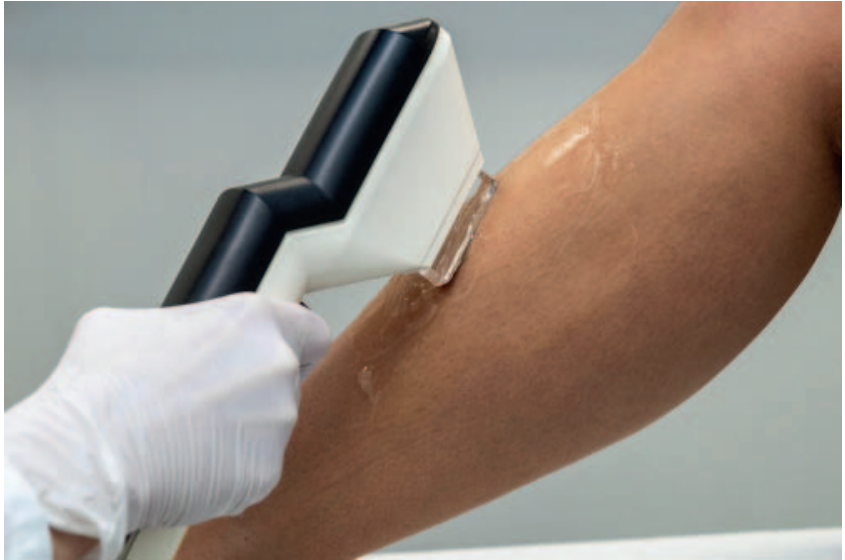

Abb. 2 Fotoepilation mittels Intense-Pulsed-Light (IPL) -Systems.

\section{Verwendete Laser und Blitzlampen}

Zur Epilation werden Laser und Blitzlampen mit Wellenlängen im roten und nahen Infrarot-Bereich (600-1100 nm) verwendet, die vorwiegend von Melanin, aber nur in geringem Maße von anderen Chromophoren der Haut absorbiert werden ( $\triangleright$ Abb. 2). Für die Epilation werden der Normal-mode-Alexandrit-Laser (755 nm), der Normal-mode-Rubin-Laser (694 nm), der gepulste Diodenlaser (800 nm und $810 \mathrm{~nm}$ ), der lang gepulste Neodymium:Yttrium-Aluminium-Granat-Laser (Nd:YAG-Laser, $1064 \mathrm{~nm}$ ) und IPL-Geräte (590-1200 nm) verwendet [5]. Neuerdings finden auch Kombinationen der erwähnten Lasersysteme Anwendung [10, 11].

Die Lichtpenetration reicht hierbei bis in die tiefen Dermisabschnitte. Es gilt: Je größer die Wellenlänge, desto größer die Eindringtiefe. Je kleiner die Wellenlänge, desto stärker die Melaninabsorption.

Vorteile der IPL-Geräte sind die niedrigeren Anschaffungskosten sowie die größere Kontaktfläche des Handstücks und damit die Möglichkeit, größere Körperflächen in kürzerer Zeit zu behandeln [5]. Anders als Laser benötigen IPL-Geräte direkten Hautkontakt und die Verwendung eines Kontaktgels zur optischen Kopplung der Strahlung mit der Haut. Da IPL-Geräte Licht unterschiedlicher Wellenlängen emittieren, können neben Melanin auch andere Chromophore wie Hämoglobin und Wasser Energie absorbieren und so ein unspezifischer Hautschaden mit Entstehung von Verbrennungen, Pigmentstörungen und Narben hervorgerufen werden.

\section{Effektivität}

Für die verfügbaren Fotoepilationsmodalitäten liegt die kurzfristige Effektivität laut Studien je nach Behandlungsparametern zwischen 30 und $70 \%$ Haarreduktionsraten nach 6 Monaten nach Beendigung der Behandlung [12]. Die Effektivität wird durch wiederholte Behandlungen gesteigert $[9,13]$.

Langzeitdaten für die Effektivität von repetitiven Behandlungen mit Alexandrit, Dioden und lang gepulstem Nd:YAG-Laser variieren erheblich. In randomisierten und nichtrandomisierten kontrollierten Studien konnte durchschnittlich eine ca. 50 \%ige Reduktion der Haare nach 6-20 Monaten nach Behandlung gezeigt werden
- Tab. 1 Auswahl absoluter und relativer Kontraindikationen.

\begin{tabular}{|l|l|}
\hline absolute Kontraindikationen & relative Kontraindikationen \\
\hline Goldsalze & Retinoide \\
\hline Lupus erythematodes & $\begin{array}{l}\text { kürzlich zurückliegende } \\
\text { Operationen im Behandlungsgebiet }\end{array}$ \\
\hline $\begin{array}{l}\text { aktive Infektionen im } \\
\text { Behandlungsgebiet }\end{array}$ & Schwangerschaft \\
\hline & Psoriasis vulgaris \\
\hline & Tattoos \\
\hline & Neigung zu Keloiden \\
\hline
\end{tabular}

[9, 12, 14-16]. Bei hellen Hauttypen (I-III nach Fitzpatrick) zeigten verschiedene Studien für Dioden-, Rubin-, Alexandrit-Laser und IPLSysteme eine vergleichbare Effektivität [16-21], wobei für IPL und Rubin-Laser insgesamt nur spärliche Langzeitdaten vorliegen [12].

\section{Patientenselektion}

Bei der Auswahl der Patienten ist neben dem Hauttyp (i. e. epidermale Melaninkonzentration), der Dicke und Farbe der Haare sowie der anatomischen Lokalisation auch auf Grunderkrankungen, insbesondere hormonelle Störungen (z. B. polyzystisches Ovarialsyndrom) oder Medikamente mit assoziiertem Haarwuchs, zu achten. Optimale Behandlungsvoraussetzungen liegen bei Patienten mit dunklen, dicken Terminalhaaren, hellem Hauttyp und unauffälligem Hormonstatus vor.

\section{Kontraindikationen}

Grundsätzlich ist die Fotoepilation ein sicheres und effektives Verfahren. Trotzdem müssen einige Kontraindikationen beachtet werden. Die Einnahme von Goldsalzen (z. B. bei rheumatoider Arthritis) ist kontraindiziert, da eine Laserbehandlung in einer Hyperpigmentierung der Haut resultieren kann. Obwohl Laserbehandlungen unter Isotretinoin-Einnahme ohne erhöhte Komplikationsraten bereits durchgeführt wurden [22, 23], stellen das Fototoxizitätspotenzial, die erhöhte Fragilität der Haut sowie die verzögerte Wundheilung und Narbenbildung unter Retinoiden eine relative Kontraindikation dar. Es wird daher eine Auswaschphase von 6-12 Monaten vor Fotoepilation empfohlen [5]. In - Tab. 1 sind ausgewählte relative und absolute Kontraindikationen aufgeführt.

\section{Patientenaufklärung}

Im Beratungsgespräch mit den Patienten ist es entscheidend, realistische Erwartungen an die Laserbehandlung zu schaffen und über die Notwendigkeit und Kosten multipler Behandlungen (mind. 4-6 Sitzungen) sowie Nebenwirkungen aufzuklären. Behandlungen erfolgen in ca. 4-wöchigen Intervallen. Eine permanente, vollständige Haarentfernung kann nicht garantiert wer- 
den, denn ein Großteil der Haare wächst wieder nach, sodass häufig regelmäßige Nachbehandlungen erforderlich sind. Eine einzelne Laserbehandlung erzielt in der Regel eine 2-6-monatige Wachstumsverzögerung [5]. Zudem ist dringend auf die Notwendigkeit von UV-Schutzmaßnahmen hinzuweisen, da eine verstärkte UV-induzierte Pigmentierung zu deutlich erhöhten Nebenwirkungsraten führt.

\section{Nebenwirkungen}

Da nicht nur der pigmentierte Haarschaft, sondern auch die Epidermis Melanin enthält und Energie derselben Wellenlänge absorbiert, besteht bei der Fotoepilation ein erhöhtes Risiko unerwünschter Nebenwirkungen an der Haut. Bei gut ausgebildeten und erfahrenen Behandlern sind die Nebenwirkungen in der Regel allerdings nur mild und transient.

Unmittelbar nach der Fotoepilation zeigt sich bei korrekten Einstellungen klinisch häufig ein für wenige Minuten bis Stunden anhaltendes perifollikuläres Ödem und Erythem [24]. Häufig werden zudem geringe bis mäßige Schmerzen während der Behandlung berichtet. Zu den seltenen Nebenwirkungen zählen Verbrennungen mit Blasen-, Krusten- und Narbenbildung sowie Hypound Hyperpigmentierungen [25, 26] ( wird der frühzeitige Einsatz von potenten topischen Glukokortikoiden empfohlen [5].

Sehr selten wurde zudem über das Auftreten von vorzeitig ergrauten oder eingewachsenen Haaren sowie inflammatorischen Veränderungen von vorbestehenden Naevi berichtet [27]. Bei ca. 0,6-10\% der Patienten, vorwiegend bei dunkleren Hauttypen mit dicken, dunklen Haaren, wurde nach Anwendung von IPL-Geräten sowie Dioden- bzw. Alexandrit-Lasern eine paradoxe Hypertrichose in behandelten und umgebenden Arealen, vor allem im Gesichtsbereich, beobachtet [5, 25, 28-30]. Als Ursache für eine Stimulation des Haarwachstums wird eine Synchronisation ruhender Haarfollikel in der Anagenphase [28] oder die Induktion einer Differenzierung von Vellushaaren zu Terminalhaaren durch eine suboptimale Energiedichte diskutiert [31]. Bei initialer Hypertrichose kann die Fotoepilation dennoch erfolgreich fortgeführt werden [5].

Akzidentelle okuläre Schäden (Retinaschädigung, Katarakt, Iritis, Uveitis, Visusfeldreduktion etc.) können durch jedes Laser- bzw. Blitzlampen-Gerät hervorgerufen werden [5]. Augenschutzmaßnahmen für Behandler und Patient in Form von Brillen oder Augenschalen, angepasst an den verwendeten Wellenlängenbereich, sind daher obligat.

Um Schmerzen und Hautschäden zu reduzieren, wird bei der Fotoepilation eine Kühlung der Haut beispielsweise durch integrierte Kontaktkühlung, Kaltluftkühlung oder Kryogenspray empfohlen. Dies gilt insbesondere für dunklere Hauttypen. Eine Kühlung der Haut und Schutz des epidermalen Melanins ermöglichen zudem die Anwendung höherer Energiedichten [32].

\section{Vor- und Nachbehandlung}

Die Haare sollten vor der Behandlung rasiert bzw. gekürzt werden, um die Rauchentwicklung und das Risiko einer Verbrennung der Haut durch aufliegende Haare zu minimieren. Mindestens 4 Wochen

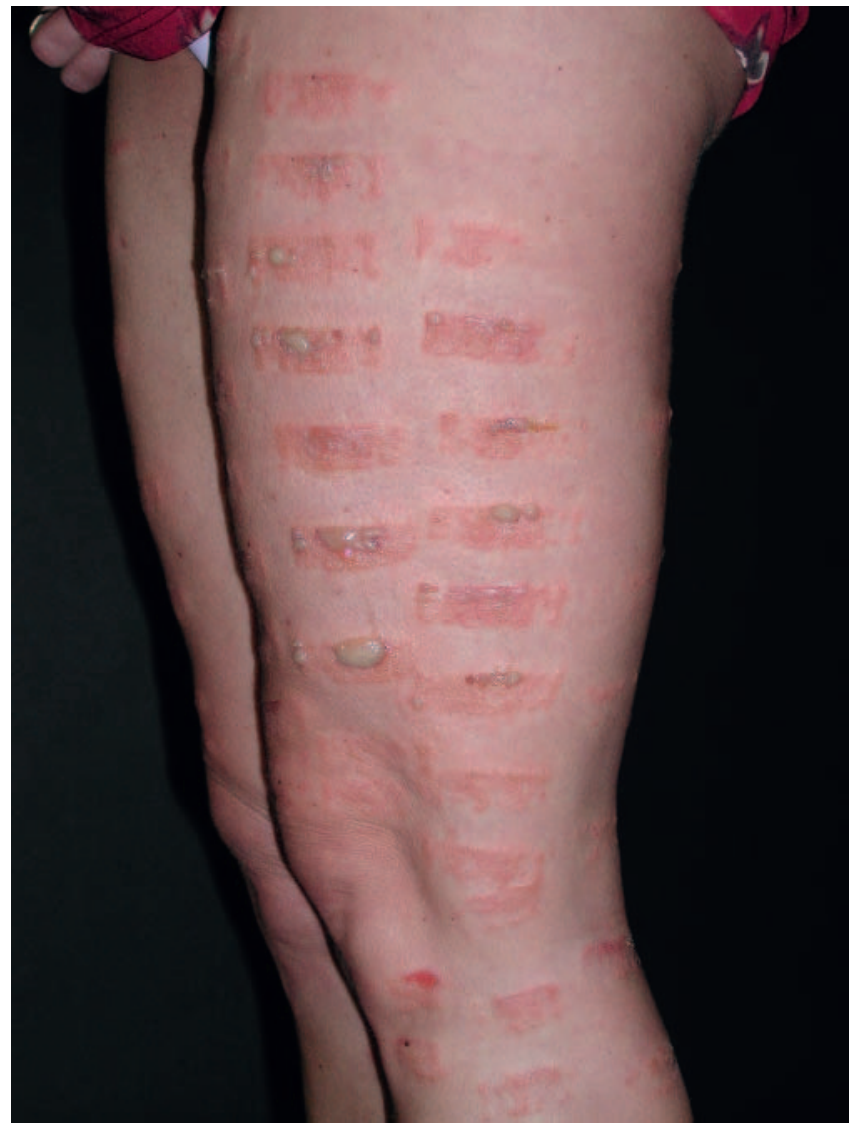

- Abb. 3 Grad-II-Verbrennung nach Fotoepilation bei unsachgemäßer Anwendung eines IPL-Systems.

vor der Behandlung sollte auf Enthaarungsmethoden mit Entfernung des Haarschaftes (z. B. Wachsen, Zupfen, Epilation) und damit des relevanten Zielchromophors Melanin verzichtet werden.

Da epidermales Melanin ebenfalls Lichtstrahlung absorbiert, ist das Risiko für Hypo- und Hyperpigmentierung, Blasen- und Narbenbildung deutlich gesteigert bei sonnengebräunter Haut. Patienten sollten daher mind. jeweils 6 Wochen vor und nach der Laserbehandlung strikten UV-Schutz betreiben (Sonnencreme mit UVAund UVB-Schutz und hohem Lichtschutzfaktor, textiler UV-Schutz, keine Sonnenbäder/Solarien). Tätowierungen sollten unbedingt bei der Behandlung ausgespart bleiben, um schwere Verbrennungen zu verhindern.

Bei schmerzempfindlichen Patienten kann ca. 1 Std. vor Behandlungsbeginn ein Lokalanästhetikum aufgetragen werden (z. B. Lidocain mit Prilocain-Creme). Nach der Behandlung führt die Kühlung der Haut beispielsweise mit Coolpacks ebenfalls zu einer Reduktion von Schmerzen und Schwellungen.

\section{Therapeutische Herausforderungen}

\section{Dunkle Hauttypen}

Aufgrund des erhöhten epidermalen Melaninvorkommens bei dunkleren Hauttypen (IV-VI nach Fitzpatrick) besteht ein deutlich erhöhtes Risiko für Nebenwirkungen bei der Fotoepilation. Bei 
dunkleren Hauttypen wird daher empfohlen, Laser bzw. IPL-Geräte mit längeren Wellenlängen, längeren Impulsdauern und konservativen Energiedichte-Einstellungen zu verwenden. Laser mit einer längeren Wellenlänge wie der Dioden (810 nm) - oder der Nd:YAG (1064 nm) -Laser werden nur in geringem Umfang von epidermalem Melanin absorbiert und verursachen daher weniger Nebenwirkungen als Geräte mit kürzeren Wellenlängen. Längere Pulsdauern erlauben eine effektive Erwärmung größerer Strukturen, wie des Haarfollikels, während kleine Strukturen, wie das epidermale Melanin, rascher abkühlen und so thermisch geschützt sind [33].

Bei Hauttypen IV bis VI konnte für den lang gepulsten Nd:YAGLaser ein effektiver Haarverlust über mind. 6 Monaten gezeigt werden, ohne Dyspigmentierung oder Verbrennungen zu verursachen [34]. Viele Experten erachten daher den lang gepulsten Nd:YAGLaser als am besten geeignet für die Haarentfernung bei dunkleren Hauttypen [5]. Eine aktuelle Metaanalyse konnte ebenfalls einen Trend zu erhöhten Haarentfernungsraten bei Laserverfahren im Vergleich zu IPL-Systemen feststellen, wobei Laser mehr akute postinterventionelle Schmerzen verursachen als IPL-Geräte [35].

\section{Helle Haare}

Helle Haare stellen nach wie vor eine große therapeutische Herausforderung dar. Blonde, graue, weiße oder rote Haare sind charakterisiert durch einen Mangel oder vollständiges Fehlen von Melanin bzw. durch Vorkommen von Eumelanin. Da für eine effektive Fotoepilation aber Melanin vorhanden sein muss, erreichen bisherige Laser oder IPL-Systeme keine zufriedenstellende Entfernung heller oder roter Haare [8, 36, 37]. Die elektrische Haarentfernung (Elektrolyse) wird daher häufig für die Entfernung heller Haare bevorzugt. Von verschiedenen Autoren wurde nun der Einsatz von kombinierten Radiofrequenz- und IPL-Geräten untersucht [38, 39]. Sadick et al. beobachteten in einem Nachbeobachtungszeitraum von 18 Monaten nach 4 Behandlungen eine Entfernungsrate von $53 \%$ bei blonden Haaren und von $44 \%$ bei weißen Haaren [39].

In einer Pilotstudie konnte durch eine fotodynamische Therapie (Off-label-Verwendung) nach 6 Monaten ein Haarverlust von $40 \%$ erzielt werden $[40,41]$. Hierbei wird eine Creme auf die Haut aufgetragen mit einem Fotosensibilisator (5-Aminolaevulinsäure), welcher sich in den Haarfollikeln anreichert und durch UV-Exposition aktiviert wird. Dies führt zur Entstehung reaktiver Sauerstoffspezies und so zur Zerstörung des Haarfollikels.

\section{Geräte für den Heimgebrauch}

Aufgrund der geringeren Kosten, des einfachen, schmerzarmen Gebrauchs und des Komforts der Eigenanwendung erfreuen sich Geräte zur Enthaarung für den Heimgebrauch wachsender Beliebtheit. Für den nichtprofessionellen Heimgebrauch werden Laser der Klasse 1 und IPL-Geräte mit niedrigeren Energiedichten von meist unter $20 \mathrm{~J} / \mathrm{cm}^{2}$ angeboten [42]. Anders als bei zugelassenen Medizinprodukten existieren für diese Art von Kosmetikprodukten in der Regel nur kleine, häufig Industrie-gesponserte, nichtkontrollierte Studien mit nur kurzen Beobachtungszeiträumen. Durchschnittlich beträgt die Reduktionsrate des Haarwachstums 40-60\% mit einem anhaltenden Effekt über ca. 3 Monate
[12, 42]. Haarentfernungsgeräte für den Heimgebrauch weisen gegenüber der professionellen Fotoepilation eine geringere Effektivität und ein kürzeres Therapieansprechen auf [12, 42]. Zudem besteht trotz integrierter Sicherheitsvorkehrungen durch unsachgemäßen Gebrauch ein Risiko für Verbrennungen und Augenschäden [5, 41]. Aufgrund des wachsenden Angebots und der zunehmenden Nachfrage durch Patienten sind Kenntnisse über die Vor- und Nachteile solcher Haarentfernungsgeräte für die Heimanwendung auch für Ärzte relevant, um Patienten kompetent beraten zu können.

\section{Laserschutz-Voraussetzungen}

Die von der Bundesregierung neuverfasste Verordnung zum Schutz vor schädlichen Wirkungen nichtionisierender Strahlung bei der Anwendung am Menschen (NiSV) trat zum 31.12.2020 in Kraft [43]. Sie gilt für Einrichtungen mit Anwendung von Laser und intensiven Lichtquellen, z. B. zur dauerhaften Haarentfernung oder zur TattooEntfernung. Anders als Tattoo-Entfernungen, welche künftig nur noch von ausgebildeten Fachärzten durchgeführt werden dürfen, können laser- und lichtbasierte Epilationsverfahren weiterhin auch durch nichtärztliche Berufsgruppen (z. B. Kosmetiker, Heilpraktiker) erfolgen, die über die erforderliche Fachkunde verfügen und eine entsprechende Schulung absolviert haben [43]. Der Gesetzgeber erkennt damit die potenziellen Gefahren bei unsachgemäßer Anwendung von Laser und Blitzlampen und fordert daher obligate Fachkenntnisse über die Grundlagen der Haut und der Strahlenquellen sowie deren potenzielle Nebenwirkungen für eine sichere und effektive, professionelle Fotoepilation.

\section{Fazit für die Praxis}

Fotoepilation mittels Laser oder IPL-Geräten stellt ein sicheres und effektives Verfahren zur Haarentfernung dar. Entscheidend ist, Patienten über den nur partiellen und temporären Haarverlust sowie die Notwendigkeit wiederholter Behandlungen aufzuklären. Auf eine ausreichende Kühlung der Haut sowie UV-Schutz sollte dringend geachtet werden. In den Händen von erfahrenen Behandlern und bei korrekten Einstellungen angepasst an die Haarbeschaffenheit und den Hauttyp treten bei der professionellen Fotoepilation nur selten schwere Nebenwirkungen wie Verbrennungen, Narben oder Pigmentverschiebungen auf. Haarentfernungsgeräte für den Heimgebrauch stellen eine kostengünstige, jedoch weniger effektive Alternative dar.

Interessenkonflikt

Die Autorinnen/Autoren geben an, dass kein Interessenkonflikt besteht.

Literatur

[1] Blume-Peytavi U, Hahn S. Medical treatment of hirsutism. Dermatol Ther 2008; 21: 329-339

[2] Kautz G, Rick K, Sandhofer M Hrsg. Photoepilation: Zur Praxis der Haarentfernung mit Licht- und Lasersystemen. Darmstadt: Steinkopff; 2004 
[3] Ross EV, Ladin Z, Kreindel M et al. Theoretical considerations in laser hair removal. Dermatol Clin 1999; 17: 333-355, viii

[4] Rogachefsky AS, Silapunt S, Goldberg DJ. Evaluation of a new superlong-pulsed 810 -nm diode laser for the removal of unwanted hair: the concept of thermal damage time. Dermatol Surg 2002; 28: 410-414

[5] Gan SD, Graber EM. Laser hair removal: a review. Dermatol Surg 2013; 39: $823-838$

[6] Goldberg DJ. Laser hair removal. In: Laser Dermatology. Pearls and problems. Oxford: Blackwell Publishing; 2007

[7] Lin TY, Manuskiatti W, Dierickx CC et al. Hair growth cycle affects hair follicle destruction by ruby laser pulses. J Invest Dermatol 1998; 111: 107-113

[8] Wanner M. Laser hair removal. Dermatol Ther 2005; 18: 209-216

[9] Lou WW, Quintana AT, Geronemus RG et al. Prospective study of hair reduction by diode laser $(800 \mathrm{~nm})$ with long-term follow-up. Dermatol Surg 2000; 26: 428-432

[10] Zerbinati N, Protasoni M, Dalla Gasperina D et al. Efficacy and safety of triple wavelength laser hair reduction in skin types IV to V. Dermatol Ther 2021; 34: e14599

[11] Raj Kirit EP, Sivuni A, Ponugupati S et al. Efficacy and safety of triple wavelength laser hair reduction in skin types IV to V. J Cosmet Dermatol 2021; 20: 1117-1123

[12] Haedersdal M, Beerwerth F, Nash JF. Laser and intense pulsed light hair removal technologies: from professional to home use. $\mathrm{Br}$ J Dermatol 2011; 165 (Suppl. 3): 31-36

[13] Toosi P, Sadighha A, Sharifian A et al. A comparison study of the efficacy and side effects of different light sources in hair removal. Lasers Med Sci 2006; $21: 1-4$

[14] Lorenz S, Brunnberg S, Landthaler M et al. Hair removal with the long pulsed Nd:YAG laser: a prospective study with one year follow-up. Lasers Surg Med 2002; 30: 127-134

[15] Davoudi SM, Behnia F, Gorouhi F et al. Comparison of long-pulsed alexandrite and Nd:YAG lasers, individually and in combination, for leg hair reduction: an assessor-blinded, randomized trial with 18 months followup. Arch Dermatol 2008; 144: 1323-1327

[16] Eremia S, Li C, Newman N. Laser hair removal with alexandrite versus diode laser using four treatment sessions: 1-year results. Dermatol Surg 2001; 27: 925-930

[17] Bouzari N, Tabatabai H, Abbasi Z et al. Laser hair removal: comparison of long-pulsed Nd:YAG, long-pulsed alexandrite, and long-pulsed diode lasers. Dermatol Surg 2004; 30: 498-502

[18] Handrick C, Alster TS. Comparison of long-pulsed diode and long-pulsed alexandrite lasers for hair removal: a longterm clinical and histologic study. Dermatol Surg 2001; 27: 622-626

[19] Dierickx CC, Grossman MC, Farinelli WA et al. Comparison between a long pulsed ruby laser and a pulsed infrared laser system for hair removal. Lasers Surg Med 1998 (Suppl. 10): 42

[20] Alster TS. Prolonged clinical experience with laser assisted hair removal: a clinical comparison of systems. Lasers Surg Med 1999 (Suppl. 11): $14-15$

[21] Nanni CA, Alster TS. Efficacy of multiple hair removal sessions using the QS Nd:YAG, long-pulsed ruby, and longpulsed alexandrite laser systems. Lasers Surg Med 1998 (Suppl. 10): 40

[22] Khatri KA. The safety of long-pulsed nd:yag laser hair removal in skin types iii-v patients during concomitant isotretinoin therapy. J Cosmet Laser Ther 2009; 11: 56-60
[23] Khatri KA, Garcia V. Light-assisted hair removal in patients undergoing isotretinoin therapy. Dermatol Surg 2006; 32: 875-877

[24] Nanni CA, Alster TS. Laser-assisted hair removal: side effects of q-switched nd:yag, long-pulsed ruby, and alexandrite lasers. J Am Acad Dermatol 1999; 41: 165-171

[25] Moreno-Arias GA, Camps-Fresneda A. Long-lasting hypopigmentation induced by long-pulsed alexandrite laser photo-epilation. Dermatol Surg 2003; 29: 420-422

[26] Alster TS, Khoury RR. Treatment of laser complications. Facial Plast Surg 2009; 25: 316-323

[27] Rasheed Al. Uncommonly reported side effects of hair removal by long pulsed-alexandrite laser. J Cosmet Dermatol 2009; 8: 267-274

[28] Alajlan A, Shapiro J, Rivers JK et al. Paradoxical hypertrichosis after laser epilation. J Am Acad Dermatol 2005; 53: 85-88

[29] Kontoes P, Vlachos S, Konstantinos M et al. Hair induction after laserassisted hair removal and its treatment. J Am Acad Dermatol 2006; 54 : 64-67

[30] Radmanesh M. Paradoxical hypertrichosis and terminal hair change after intense pulsed light hair removal therapy. J Dermatolog Treat 2009; 20: 52-54

[31] Lolis MS, Marmur ES. Paradoxical effects of hair removal systems: a review. J Cosmet Dermatol 2006; 5: 274-276

[32] Raulin C, Greve B, Hammes S. Cold air in laser therapy: first experiences with a new cooling system. Lasers Surg Med 2000; 27: 404-410

[33] Raulin C, Greve B. Aktueller Stand der Photoepilation. Der Hautarzt 2000; 52: 809-817

[34] Ismail SA. Long-pulsed Nd:YAG laser vs. intense pulsed light for hair removal in dark skin: a randomized controlled trial. Br J Dermatol 2012; 166: 317-321

[35] Dorgham NA, Dorgham DA. Lasers for reduction of unwanted hair in skin of colour: a systematic review and meta-analysis. J Eur Acad Dermatol Venereol 2020; 34: 948-955

[36] Bouzari N, Tabatabai H, Abbasi Z et al. Hair removal using an 800-nm diode laser: comparison at different treatment intervals of 45,60 , and 90 days. Int J Dermatol 2005; 44: 50-53

[37] Lorenz S, Brunnberg S, Landthaler $M$ et al. Hair removal with the longpulsed Nd: YAG laser: a prospective study with one year follow-up. Lasers Surg Med 2002; 30: 127-134

[38] Sadick NS, Shaoul ]. Hair removal using a combination of conducted radiofrequency and optical energies - an 18-month follow-up. J Cosmet Laser Ther 2004; 6: 21-26

[39] Sadick NS, Laughlin SA. Effective epilation of white and blond hair using combined radiofrequency and optical energy. J Cosmet Laser Ther 2004; 6: 27-31

[40] Dierickx CC, Grossman MC. Laser Hair Removal. In: Dover JS, Goldberg DJ, Hrsg. Laser and Lights. Volume 2 Philadelphia: Elsevier Saunders; 2005: 61-76

[41] Shin H, Yoon JS, Koh W et al. Nonpigmented hair removal using photodynamic therapy in animal model. Lasers Surg Med 2016; 48: 748-762

[42] Hession MT, Markova A, Graber EM. A review of hand-held, home-use cosmetic laser and light devices. Dermatol Surg 2015; 41: 307-320

[43] Kautz G, Rick K, Sandhofer M Hrsg. Photoepilation: Zur Praxis der Haarentfernung mit Licht- und Lasersystemen. Darmstadt: Steinkopff. 2004 